\title{
Registrazione di terremoti vicini con sismometri a lungo periodo
}

(Recording of near earthquakes by long-period seismometers)

\author{
E. Mantovani (*)
}

Ricevuto il 10 Giugno 1972

Riassunto. - Vengono riportate le caratteristiche della nuova rete a lungo periodo in Italia e la possibilità di sfruttare i sismometri della rete per la registrazione dei sismi vicini.

SUmmaRY. - The characteristics of the long-period seismic network in Italy are described and a method to use the long-period seismometer for the recording of near earthquakes is suggested.

L'Istituto di Geofisica dell'Università di Bologna, in collaborazione con le Università di Karlsruhe e Ucla di Los Angeles, sta svolgendo una ricerca sulla crosta del Mediterraneo con particolare riguardo al bacino del Tirreno.

Questa ricerca è condotta col metodo basato sulla dispersione delle onde superficiali, per la registrazione delle quali è stata installata una rete di stazioni a lungo periodo $\left(^{4}\right)$ (Fig. 1, Tabella 1).

Una serie di prove ha permesso di mettere a fuoco prima e superare poi i problemi connessi con la realizzazione di una stazione sismica a corto periodo che utilizza al posto dei normali pendoli a corto i sismometri a lungo periodo della citata rete.

(*) Istituto di Geofisica. Università di Bologna (Italia). 


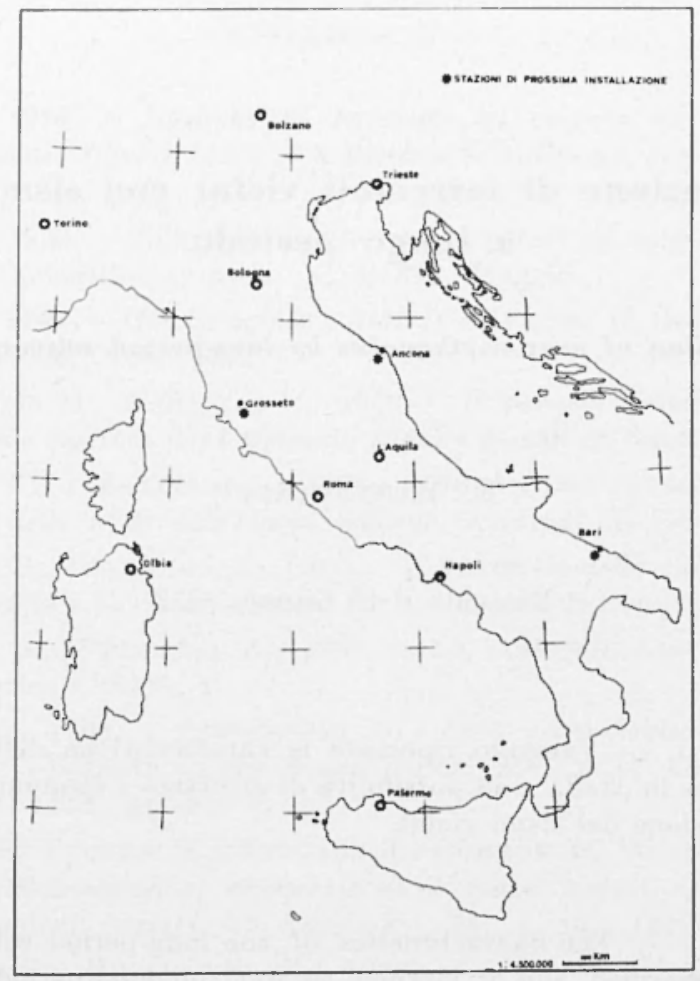

Fig. 1 - Rete sismica a lungo periodo in Italia.

Tabella 1

\begin{tabular}{|c|c|c|c|c|c|c|c|}
\hline ataxione & $\begin{array}{c}\text { perlodo } \\
\text { sismormatro } \\
(\cdots+)\end{array}$ & $\begin{array}{l}\text { periodo } \\
\text { ontvanometro } \\
(m)\end{array}$ & $\begin{array}{l}\text { emorzam. } \\
\text { sism. }\end{array}$ & $\begin{array}{l}\text { emorzam. } \\
\text { galv. }\end{array}$ & $\begin{array}{l}\text { massima } \\
\text { ampliticaxione } \\
\text { dinamica }\end{array}$ & 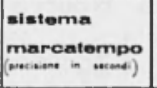 & $\begin{array}{l}\text { velooita di } \\
\text { regintraxiono } \\
\text { (-m/ntionte) }\end{array}$ \\
\hline Bologna & 13 & 100 & ertitioo & critico & 483 & $0,011^{A}$ & 30 \\
\hline Bolxano & 15 & 100 & eritico & erltico & 355 & $0,011^{B}$ & 30 \\
\hline Napoll & 15 & 97 & crutico & crittico & 348 & $0,01 \quad$ & 30 \\
\hline Olbin & 15 & 116 & arltico & eritico & 435 & $0,01 \quad$ & 30 \\
\hline Palermo & 15 & 95 & critico & erblico & 410 & $0,01 \quad$ & 30 \\
\hline Torino & 15 & 100 & eritico & crtlico & 363 & 0,01 & 30 \\
\hline
\end{tabular}

A - stazione oraria Pateck Philippe; B - ricevitore T75. 
La scelta degli strumenti di una stazione sismica a corto periodo è condizionata dalle seguenti esigenze:

a) Massima sensibilità in corrispondenza delle frequenze dove ¿̀ generalmente concentrata l'energia delle onde $P(0,5 \div 1,5$ sec $)$.

Questo favorisce una salita ripida del primo impulso e di conseguenza precisione nella determinazione del tempo di arrivo.

b) Discesa ripida dell'amplificazione sia per i periodi $>3$ sec, sia per quelli $<0,6$ sec. La prima, per evitare il disturbo di tutta l'energia sismica a lungo periodo (onde $S$ e superficiali), ma soprattut to per ridurre al minimo quello microsismico $(3 \div 7 \mathrm{sec})$ dovuto a perturbazioni atmosferiche. La seconda per evitare l'altro tipo di attività microsismica a più alta frequenza $(0,4 \div 0,6 \mathrm{sec})$ causata da disturbi di carattere locale (traffico, industrie...).

c) Minor ritardo possibile tra l'arrivo del segnale e la sua registrazione.

Le condizioni $a$ ) b) c) non sono realizzabili contemporaneamente. Infatti per ottenere un ritardo minimo per le frequenze delle onde $P$ sarebbe necessario usare galvanometri e sismometri di periodo rispettivamente $\ll 1$ sec e $\gg 1$ sec, il che contrasta con i punti $a$ ) e $b$ ), che come si può vedere dalle curve di risposta delle figg. 2, 3, portano ad una scelta di $T_{g}$ e $T_{s}$ compresi tra 0,5 e 1,5 secondi.

Generalmente viene dato maggior peso ai primi due punti cercando di limitare il ritardo al minimo possibile.

La scelta del periodo degli strumenti va fatta stazione per stazione in base ai valori della contingente attività microsismica.

Stazione a CORTo PERIOdo CON SISMOMETRo DI $T_{s}=15$ SEC E GALVANOMETRO DI $T_{\theta}=1,8$ SEC.

Analizziamo ora la possibilità di ottenere le caratteristiche precedenti con un sistema che al posto di un pendolo di $0,5 \div 1,5$ sec, ne impieghi uno di 15 secondi.

L'inconveniente legato all'uso di un tale tipo di sismometro è lo scarso filtraggio dei periodi lunghi (Fig. 2).

Per eliminare o per lo meno attenuare la difficoltà bisogna ricorrere ad un filtro elettronico che può essere sia di tipo attivo, cioè com- 


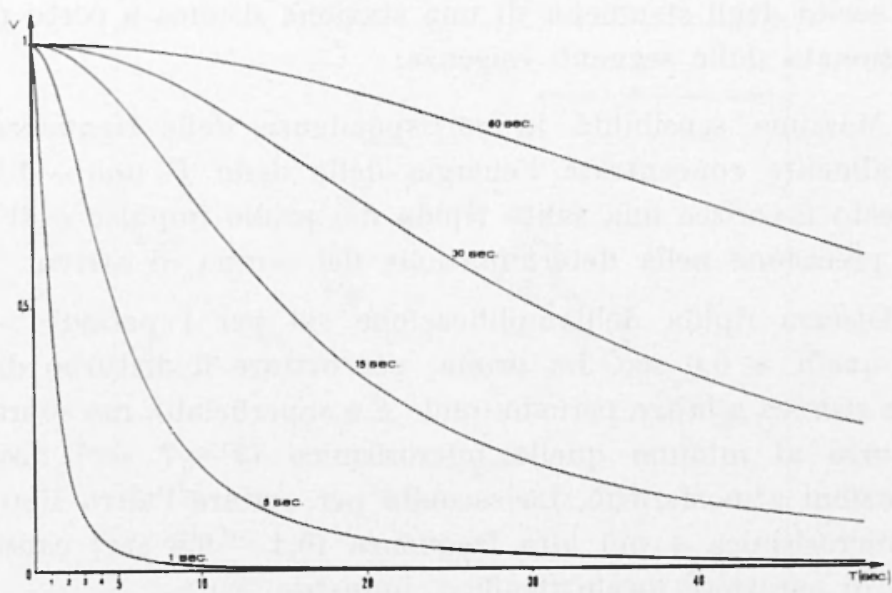

Fig. 2 - Curve di risposta del sismometro, criticamente smorzato, per diversi valori del periodo.

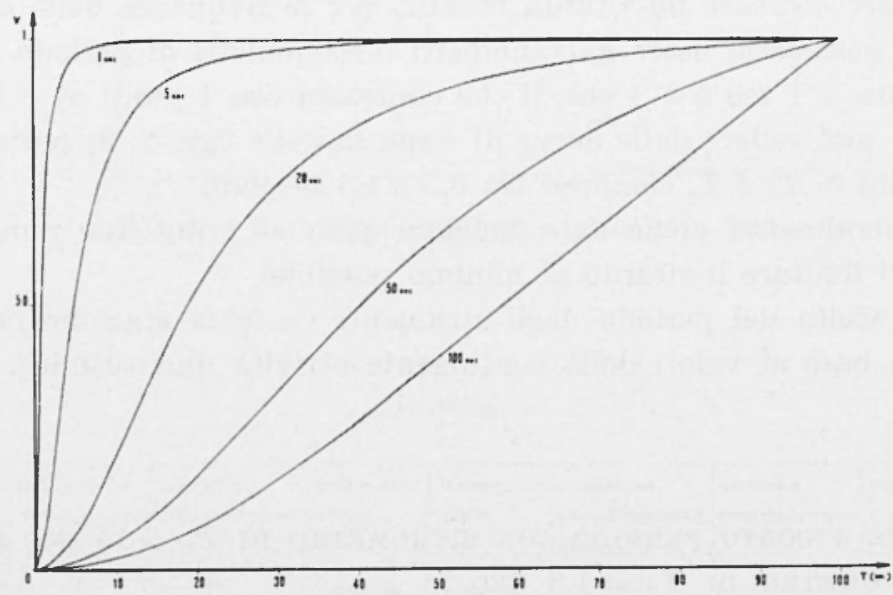

Fig. 3 - Curve di risposta normalizzate del galvanometro criticamente smorzato.

posto di elementi amplificatori, sia di tipo passivo, composto di soli condensatori, induttanze, resistenze.

Col primo tipo è possibile ottenere, oltre ad una amplificazione del segnale, anche un taglio molto netto alle frequenze volute: esiste 
tuttavia il problema, non sempre risolvibile, dell'eliminazione del rumore elettronico.

In attesa di perfezionare un filtro attivo alatto allo scopo si ì ricorso ad un semplicissimo filtro passa-alto di tipo CR (Fig. 4).

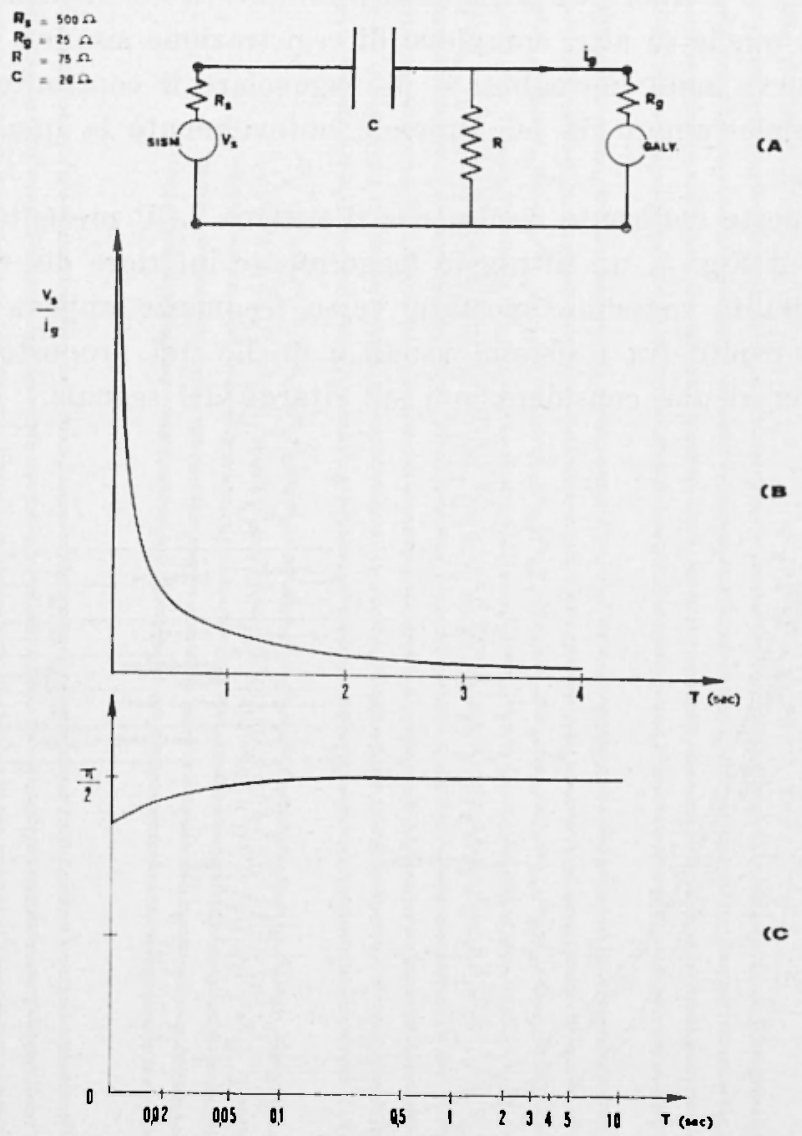

Fig. 4 - A) schema del filtro ( $R$ e sua risposta in ampiezza B) e fase $C)$.

Il sistema in questione, che per comodità è stato chiamato LCP, viene cosi arl essere composto da:

1) Sismometro a lungo periodo tipo Sprengnether S-5007- $\mathrm{V}$

$$
\begin{aligned}
\text { massa } & =11,2 \mathrm{~kg} \\
\text { sensibilità } & =89 \mathrm{~V} / \mathrm{m} / \mathrm{sec} \\
\text { periodo } & =15 \mathrm{sec} .
\end{aligned}
$$


2) Galvanometro a corto periodo tipo Cambridge $41127 / 1$

$$
\begin{aligned}
\text { sensibilità } & =300 \mathrm{~mm} / \mu \Lambda \\
\text { periodo } & =1,8 \mathrm{sec} .
\end{aligned}
$$

3) Filtro passivo costituito da una singola cella CR.

In Fig. 5 è riportata la risposta in ampiezza del sistema in esame accanto a quelle di altri complessi di registrazione sismica.

Le curve sono normalizzate per agevolare il confronto del rapporto segnale-rumore da cui dipende notevolmente la qualità di un sismografo.

Da questo confronto risulta che il sistema I,CP presenta, rispetto agli altri di Fig. 5, un filtraggio leggermente inferiore dei microsismi e la sensibilita massima spostata verso frequenze appena più alte.

Il confronto fra i sistemi usuali e quello qui proposto va completato con alcune considerazioni sul ritardo del segnale.

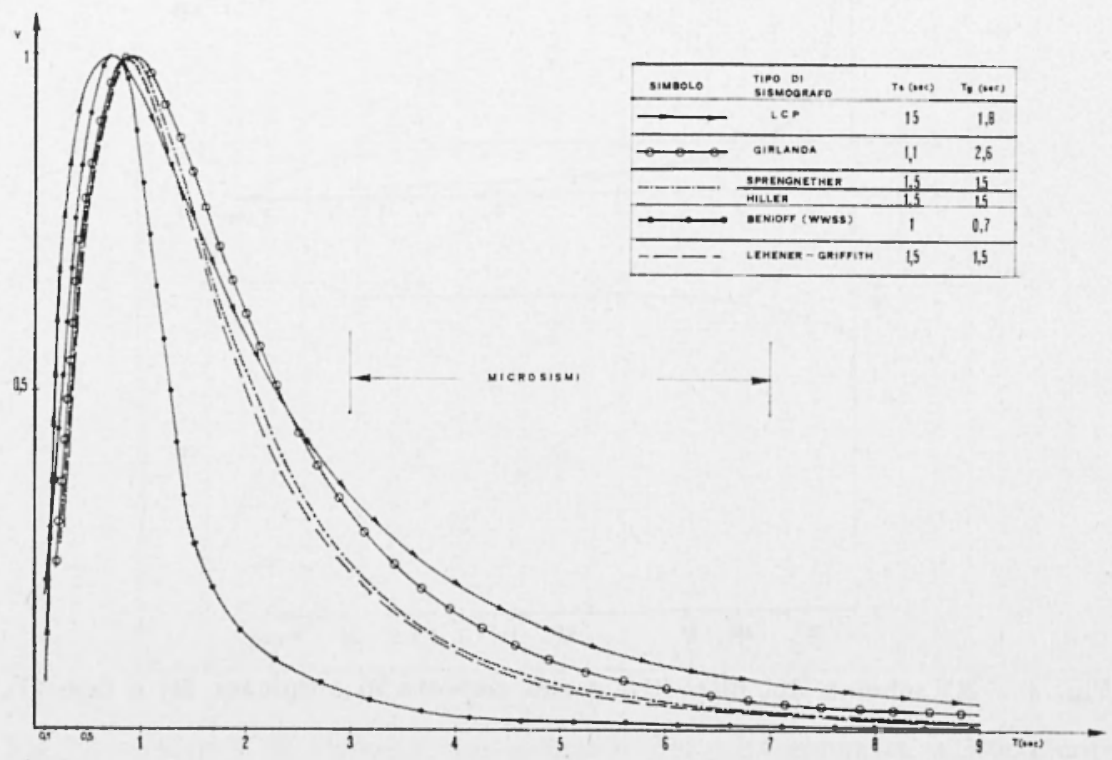

Fig. 5 - Curve di risposta dei vari tipi di sismometri elettomagnetici confrontate con quella del sistema LCP.

Una normale stazione a corto periodo, che per semplicità consideriamo rappresentata da un sistema con $T_{s}=1$ sec e $T_{s}=1$ sec, presenta un ritardo tra l'arrivo delle onde $P$ e la loro registrazione 
REGISTRAZIONE DI TERREMOTI VICINI CON SISMOMETRI A LUNGO PERIODO 557

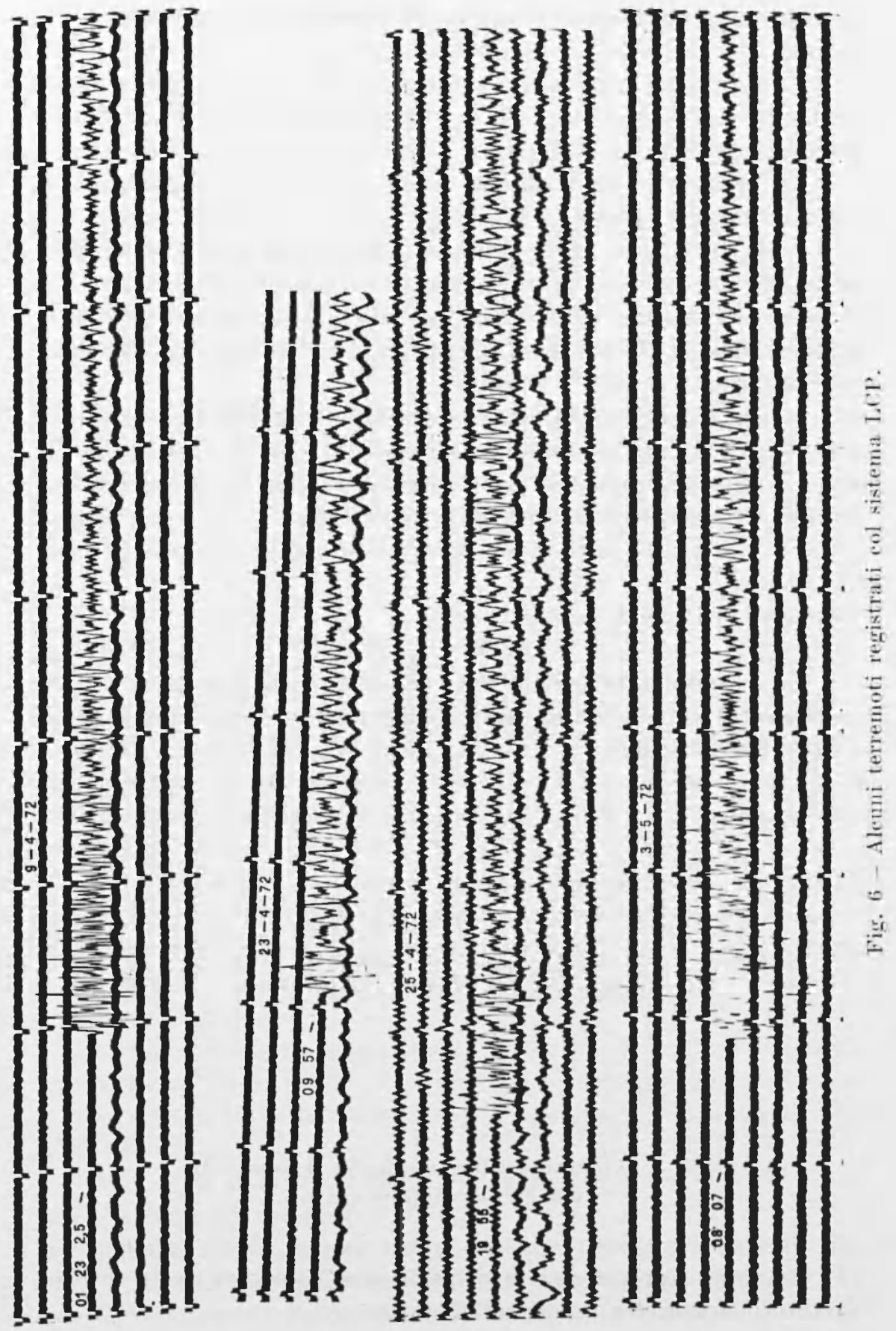


di circa mezzo periodo ( $\pi / 2$ dovuto al sismometro e $\pi / 2$ al galvanometro).

Il sistema L(P pur essendo composto da strumenti di caratteristiche diverse (sismometro e filtro) dal precedente provoca un ritardo pressoché analogo.

Per quanto riguarda il ritardo del galvanometro l'analogia tra i due sistemi è evidente.

Il sismometro a lungo periodo del sistema LCP, invece, si differenzia da quello $\mathrm{CP}$ in quanto la sua maggiore inerzia gli permette di ridurre al minimo il ritardo tra l'evento e la sua registrazione. L'unico effetto è il ribaltamento del segnale, che presenterà quindi massimi al posto di minimi e viceversa.

La quasi assenza di ritarlo dovuto al sismometro viene però compensata da quello introdotto dal filtro. Infatti per frequenze di $\sim 1$ sec, l'impedenza è quasi puramente reattiva e provoca un ritardo tra tensione e corrente di circa $\pi / 2$ (Fig. 4).

\section{STAZIONE A CORTO E LLNGo PERIODO}

La seconda parte dell'esperimento consiste nell'impiego di un solo sismometro con periodo di $15 \mathrm{sec}$ per la registrazione simultanea dei corti e lunghi periodi.

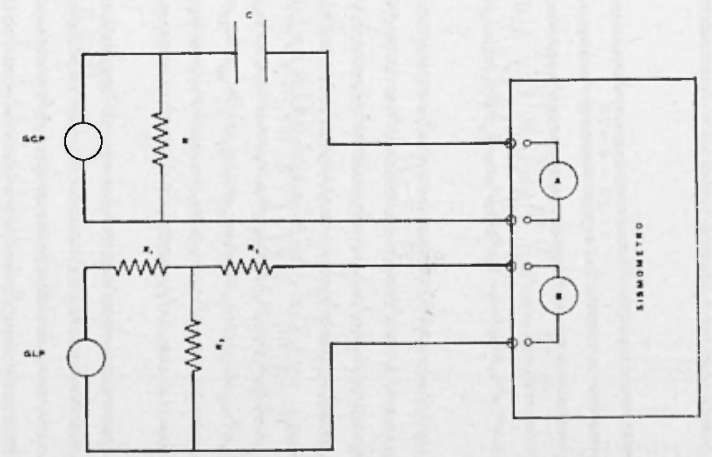

Fig. 7 - Circuiti di collegamento tra il sismometro e i due galvanometri $\mathrm{G}_{L P}$ e $\mathrm{G}_{C P}$.

$\Lambda$ questo scopo si sono sfruttate le due bobine di uscita di cui ì fornito il sismometro Sprengnether S-5007-V. 
Un segnale è stato normalmente inviato al galvanometro IP $\left(T_{0}=100 \mathrm{sec}\right)$ e l'altro al galvanometro $\mathrm{CP}\left(T_{g}=1,8 \mathrm{sec}\right)$. Il problema principale è quello di evitare qualsiasi tipo di interazione tra i due complessi.

Le uscite sono elettricamente indipendenti ed è perciò da escludere un disturbo diretto tra i due circuiti.

L'unica possibilità di interazione rimane quindi nella reazione che ogni galvanometro può avere sul penclolo.

Su questo argomento, esistono già lavori teorici $\left({ }^{1}\right)$ in cui si chiarisce che per valori della massa superiore a $7 \mathrm{~kg}$ la reazione del galvanometro sul pendolo si può considerare trascurabile a tutti gli effetti pratici.

Per aggiungere alle previsioni teoriche una conferma sperimentale, si è procerluto al confronto delle curve di calibrazione ottenute con $\mathrm{i}$ due galvanometri collegati sia singolarmente che contemporaneamente al sismometro, col risultato di trovarle sovrapponibili entro le fluttuazioni prodotte da microsismi, errori di osservazione ecc.

\section{CONFRONTO TRA LE CURVE DI CALIBRAZIONE}

La verifica che il complesso a lungo periorlo non viene influenzato dal collegamento del galvanometro $\mathrm{CP}$ al sismometro, è fatto mediante il confronto tra le due curve di calibrazione che si ottengono nelle condizioni di collegamento rappresentate in Fig. 8 dagli schemi A) B).

La presenza però di una serie di disturbi aleatori come microsismi, derive termiche ecc. rende poco significativo un confronto diretto tra le due curve. Si è pensato perciò di determinare per la condizione A) un parametro rappresentativo della situazione media e rispettivo campo di variabilità.

Si sono ricavate e digitalizzate 10 curve di calibrazione nella condizione A). Si è ottenuta una serie di 45 coefficienti di correlazione provenienti da tutte le combinazioni di confronti tra le curve digitalizzate.

Da questa serie si è oftenuto un valore merlio $C=0,998$ e uno scarto quadratico medio $\xi=0,001$.

Si è poi correlata una curva ottenuta nella condizione B) con tutte le 10 ottenute nella condizione $A$ ) ricavando 10 coefficienti che coincidevano tutti, entro lo searto $\overline{\bar{\xi}}$, con il valor medio $\bar{C}$. 
Ripetuto questo procedimento per altre quattro curve di B) e ottenuti, tranne 2 su 10 coefficienti, gli stessi risultati precerlenti si è dedotto che il complesso a lungo periorlo non sente la presenza del galvanometro CP.
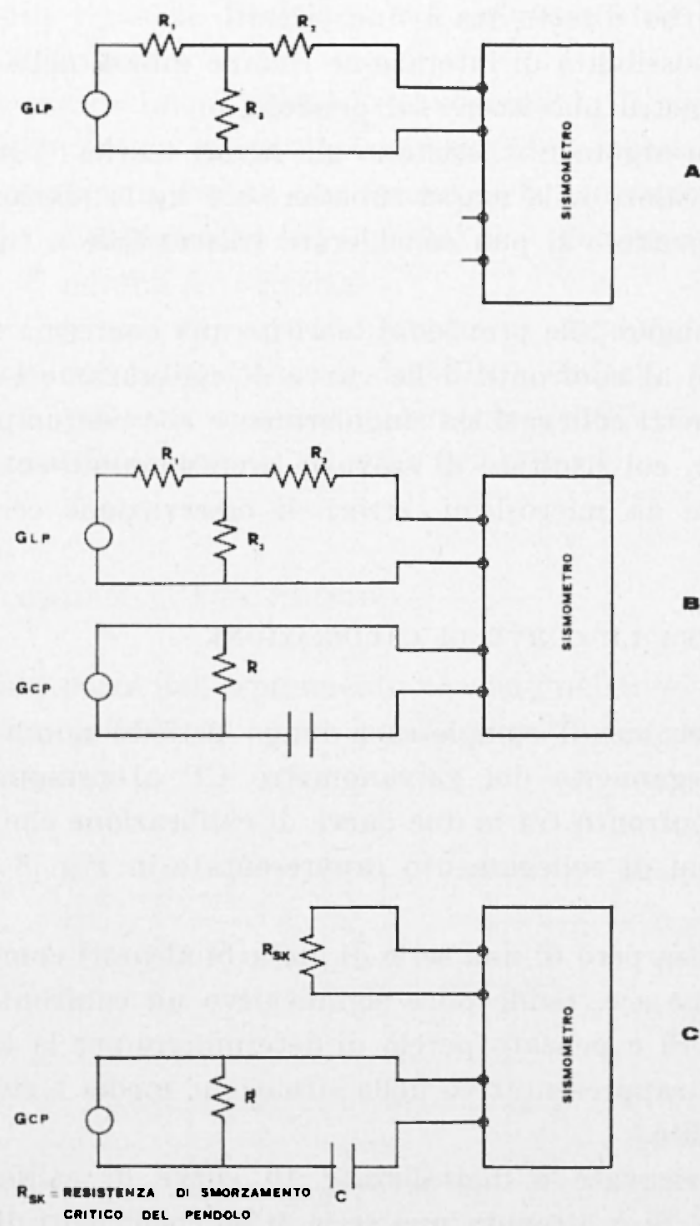

Fig. 8 - A) Complesso a lungo periodo; B) entrambi i complessi (LP.CP) in funzione contemporaneamente: C) complesso a corto periodo.

Lo stesso metodo condotto con le curve ottenute nelle condizioni B) e C) ha escluso infine qualsiasi trasferimento apprezzabile di energia dal galvanometro LP a quello CP. 


\section{Circuiti Di COLJ.eG.Miento}

Un corretto funzionamento degli strumenti richiede che:

1) $\mathrm{I}$ due galvanometri e sismometri siano vicini allo smorzamento critico.

2) L'attenuazione sia del complesso CP che LP sia tale da tenere il livello dei microsismi, nelle registrazioni, inferiore a $1.2 \mathrm{~mm}$.

In Fig. 7 sono riportati i circuiti richiesti dalle condizioni precedenti.

La connessione a $T$ del lungo periodo produce lo smorzamento critico sia del pendolo che del galvanometro LP e la attenuazione necessaria.

Il circuito di accoppiamento del corto periodo deve produrre lo smorzamento critico del galvanometro CP e la giusta attenuaione del segnale. Inoltre deve presentare al sismometro un'impedenza molto maggiore della sua resistenza di smorzamento critico per evitare un sovrasmorzamento del pendolo.

\section{CONCLUSIONI}

Le considerazioni precedenti permettono di trasformare le attuali stazioni a lungo periodo in stazioni a doppia registrazione, col risultato di creare una rete a corto periodo che permetterebbe un notevole miglioramento nell'accuratezza con cui vengono determinati i parametri sismici dei terremoti vicini e, soprattutto, di quelli che sfuggono alle basse sensibilità dei vecchi sismografi Wieckert.

Per la trasformazione è sufficiente completare ogni stazione con un galvanometro $\mathrm{CP}$ di alta sensibilità e con un registratore fotografico con velocità di rotazione del tamburo di $6 \mathrm{~cm} /$ minuto.

L'accuratezza del tempo è garantita dagli orologi a quarzo (sincronizzati via radio con una stazione emittente svizzera) di cui sono ormai corredate quasi tutte le stazioni della rete.

\section{SVILUPPI}

I risultati conseguiti fino ad ora non sono che un primo passo dell'esperimento in corso e rimangono quindi sotto esame alcuni aspetti del nuovo sistema che potrebbero essere passibili di miglioramento. 
Il principale, come già visto, ì il filtraggio dei microsismi, il quale pur essendo già quasi allo stesso livello degli altri sistemi per, noi non i ancora del tutto soddisfacente.

A questo scopo si stanno studiando altri tipi di filtri capaci di fornire tagli più netti senza introdurre sensibili ritardi del segnale.

$V i$ ì inoltre la possibilità di collegare al sismometro IJP una serie di galvanometri con diverso periodo. Si otterrebbe cosi un insieme di complessi sensibili a intervalli diversi di frequenze, interessanti diversi campi di studio come: sismicità locale $(0,1 \div 1,5$ sec $)$, microsismi $(2 \div 8$ sec), onde superficiali (oltre 10 sec) ecc.

\section{RivgRaziamevTo}

Ringrazio il Prof. M. Caputo per le fruttuose discussioni ed i preziosi consigli.

\section{BIBIAOOGRAFIA}

(1) CHAKRABART S. K., 1949. -- Response characteristics of electromagnetic seismographs and their dependance on the instrumental constants. "Bull. Seism., Soc. Am.", 39. 205-218.

(2) Eaton J. P., 1957. - Theory of electromagnetie seismographs. "Bull. Seism., Soc. Am. ", 47, 37.75.

$\left({ }^{3}\right)$ Nutti. O., McEvilsy V., 1961. - The response characteristics of long period seismographs of the Saint Louis Eniversity network. Earthquake Notes, sept.-Dec., 1961.

(4) Press F.. Ewing M., Jenner F., 1958. -- A long period Seismograph system. "Trans. Am. Geophys. Union ", 38, 106-108.

(5) Wenner F., 1925. - New Seismometer equipped for Electromagnetic damping and electromagnetic and optical magnification. Bureau of Stand., "Journ. of Res, ", 2. 962-999, Res. Paper No 66. 